\title{
Respiratory Motion Correction in Dynamic PET with a Single Attenuation Map
}

\author{
Elise C. Emond, Student Member, IEEE, Alexandre Bousse, Maria Machado, Joanna Porter, Kjell Erlandsson, \\ Ashley M. Groves, Brian F. Hutton, Senior Member, IEEE and Kris Thielemans, Senior Member, IEEE
}

\begin{abstract}
In addition to static tracer uptake values used routinely in clinical facilities, PET imaging can provide useful information on tracer kinetics via the use of dynamic acquisitions where a set of time frames are acquired starting from the injection/inhalation of the radiotracer. In lung studies, kinetic parameters, estimated from compartmental modelling, are however affected by respiratory motion. When only one attenuation image is available, most existing motion compensation strategies are not appropriate for the initial short time frames, especially as the activity distribution changes rapidly over the early part of the dynamic acquisition. This work presents a preliminary study to handle respiratory motion using a two-step process that uses gated dynamic data as input. We first use joint reconstruction of activity and motion on the entire gated PET data to estimate deformation fields. This allows the subsequent reconstruction of each time frame separately with motion compensation. We present results comparing on one hand the compartment model fit residuals with and without respiratory motion compensation and on the other hand the diaphragm position in non-attenuation corrected images and from this method.
\end{abstract}

\section{INTRODUCTION}

$\mathbf{P}$ OSITRON Emission Tomography (PET) is a powerful tool to assess metabolism using a radiolabelled tracer. In addition to providing metabolic uptake when the tracer distribution has stabilised ("static" acquisitions), PET can be used to assess kinetic processes (e.g., blood flow and metabolic binding rates). Compartment models are often used to extract such "kinetic parameters" [1] but their use is impeded in chest PET acquisitions, where non-negligible cardiac and respiratory motion occurs and gated reconstructions are not possible for the initial short frames (sometimes less than $5 \mathrm{~s}$ ).

This work aims to show the possibility of applying a Joint PET Reconstruction and Motion Estimation (JRM) [2], [3], in order to estimate the motion between gates for the entire dynamic acquisition (i.e., summing time frames). The dynamic respiratory-gated frames are then reconstructed with motion compensation. The model also accounts for the motion of the

Manuscript received December 13, 2019. This work was supported by GlaxoSmithKline (BIDS3000030921). Alexandre Bousse was funded by GE Healthcare. Research was supported by NIHR UCLH Biomedical Research Centre.

E. C. Emond is with the Institute of Nuclear Medicine, University College London, London NW1 2BU, UK (e-mail: elise.emond.16@ucl.ac.uk).

M. Machado, A. M. Groves, B. F. Hutton and K. Thielemans are with the Institute of Nuclear Medicine, University College London, UK.

A. Bousse was with the Institute of Nuclear Medicine, University College London, London NW1 2BU, UK but is now with LaTIM, INSERM, UMR 1101, Université de Bretagne Occidentale, Brest, France.

J. Porter is with the Centre for Inflammation and Tissue Repair, University College London, and with the Interstitial Lung Disease Centre, University College London Hospital, UK. attenuation map to prevent attenuation/activity misalignment. We then apply compartment modelling to the reconstructed time frames. The motion estimation from the joint reconstruction is validated against diaphragm position in non-attenuation corrected (AC) gated images and CINE-CT images (when available). The kinetic results are assessed by comparing the compartment model fit residuals of the presented method and of standard OSEM reconstructions.

\section{THEORY}

We assume that the data can be gated according to a surrogate signal, where the deformation between the gates does not depend on time. We can then first estimate the motion and subsequently tracer kinetics.

a) Motion Estimation: The listmode data from the entire dynamic acquisition is respiratory gated, assuming that the deformation between gates does not depend on time. The first part of the acquisition is however discarded to estimate the motion, in order to avoid problems with fast kinetics after injection (the duration can be determined by plotting the number of counts versus acquisition time). The acquired PET data $\boldsymbol{g}=\left\{\boldsymbol{g}_{\ell}^{m}\right\}_{(\ell, m) \in \llbracket 1, n_{\mathrm{g}} \rrbracket \times \rrbracket 1, n_{\mathrm{f}} \rrbracket}$ (where $n_{\mathrm{g}}$ denotes the number of gates and $n_{\mathrm{f}}$ the number of time frames) is binned into a collection of random vectors $\boldsymbol{g}_{\ell}^{m}$, each following a Poisson distribution:

$\forall(\ell, m) \in \llbracket 1, n_{\mathrm{g}} \rrbracket \times \llbracket 1, n_{\mathrm{f}} \rrbracket, \boldsymbol{g}_{\ell}^{m} \sim \operatorname{Poisson}\left(\overline{\boldsymbol{g}}_{\ell}^{m}\left(\boldsymbol{f}^{m}, \varphi_{\ell}, \boldsymbol{\mu}\right)\right)$, where $\overline{\boldsymbol{g}}_{\ell}^{m}$ is the expected number of counts at gate $\ell$ and frame $m$,

$$
\left[\overline{\boldsymbol{g}}_{\ell}^{m}\left(\boldsymbol{f}^{m}, \varphi_{\ell}, \boldsymbol{\mu}\right)\right]_{i}=\tau_{\ell, m} a_{i}\left(\mathcal{W}_{\varphi_{\ell}} \boldsymbol{\mu}\right) H_{i} \mathcal{W}_{\varphi_{\ell}} \boldsymbol{f}^{m}+s_{i, \ell, m},
$$

depending on the activity image $\boldsymbol{f}^{m}$ at a time frame $m$, the attenuation image $\boldsymbol{\mu}$ and $\varphi_{\ell}$ a deformation field corresponding to the gate $\ell . \mathcal{W}_{\varphi \ell}$ is the warping operator defined by the deformation $\varphi_{\ell}, \tau_{\ell, m}$ is the gate/frame duration, $a_{i}\left(\mathcal{W}_{\varphi_{\ell}} \boldsymbol{\mu}\right)$ is the attenuation term along the line of response corresponding to the bin $i$ and $H_{i}$ is the detection bin system response and $s_{i, \ell, m}$ is the background term. Note that in this model $\boldsymbol{f}^{m}$ and $\boldsymbol{\mu}$ are affected by the same motion.

For computational reasons, the gated data are summed over the frame index $m$, forming $\boldsymbol{g}_{\ell}=\sum \boldsymbol{g}_{\ell}^{m}$. We denote $\boldsymbol{\varphi}=$ $\left\{\varphi_{\ell}\right\}_{\ell \in \llbracket 1, n_{\mathrm{g}} \rrbracket}$. The method uses the implementation of JRM in Bousse et al. [3] as for a static acquisition, resulting in the following optimisation problem:

$$
(\hat{\boldsymbol{f}}, \hat{\boldsymbol{\varphi}}) \in \arg \max _{\boldsymbol{f}, \boldsymbol{\varphi}} \Phi(\boldsymbol{f}, \boldsymbol{\varphi}, \boldsymbol{\mu}) .
$$






Fig. 1. Overview of the method

where $\Phi(\boldsymbol{f}, \boldsymbol{\varphi}, \boldsymbol{\mu})$ is the (penalised) log-likelihood, using a Bspline parametrisation of the deformation fields. Here $\hat{f}$ is an average motion-compensated image, which will not be used as we are only interested in the motion $\hat{\varphi}$ for the next step.

b) Dynamic Reconstructions: Using the estimated deformation $\hat{\varphi}$, Motion-Compensated Image Reconstruction (MCIR) is performed for each time frame. The output is the estimated motion-compensated activity images $\left\{\hat{\boldsymbol{f}}^{m}\right\}_{m}$ at each frame $m$.

c) Compartmental Modelling: A compartment model [1] is used to estimate kinetic parameters from the previous dynamic MCIR reconstructions. The lung tissue tracer concentration $C(t)$ derived from a reversible 1-tissue compartment model (accounting for time delays) is given by:

$C(t)=V_{\mathrm{B}} C_{\mathrm{P}}(t-\mathrm{d} t)+\left(1-V_{\mathrm{B}}\right) K_{1} \exp \left(-k_{2} t\right) \otimes C_{\mathrm{P}}(t-\mathrm{d} t)$

where $C_{\mathrm{P}}(t)$ is the plasma tracer concentration at an instant $t, \mathrm{~d} t$ is the time delay between the arrival of the tracer in the blood and in the lung, $K_{1}$ and $k_{2}$ are the kinetic parameters. In this work, $C_{\mathrm{P}}$ is approximated with $C_{\mathrm{B}}$, the whole blood tracer concentration (using an image-derived input function from a region of interest drawn on the ascending aorta).

d) Method Representation: See Figure 1.

\section{EXPERIMENTS AND RESULTS}

\section{A. Patient Data}

11 patients underwent either a CTAC or CINE-CT acquisition, followed by a dynamic PET acquisition $(12 \times 5$ s, $12 \times 10 s, 6 \times 20$ s, $5 \times 60$ s, $2 \times 120$ s) on a GE Discovery 710 scanner [4] using monitoring with a Varian RPM system. The entire listmode files were unlisted into 5 respiratorygated projection data based on the RPM displacement. When available, the CINE-CT acquisition was binned into 5 respiratory-gated images. Similarly as in [5], the first minute of the acquisition was skipped for the motion estimation. An ungated time of flight (TOF) OSEM reconstruction of the entire data was used as a first guess of $\hat{f}$ in JRM. Following that 6 alternations of motion estimation and MCIR were performed on the entire dataset to estimate the deformation fields $\hat{\varphi}$. The gated dynamic data were then reconstructed separately using an in-house implementation of TOF MCIR (6 iterations, 8 subsets) using GE projectors and background sinograms. Finally a reversible 1-tissue compartment modelto estimate blood volume fractions $V_{\mathrm{B}}[6]$-was fitted, within a dilated lung mask, to the dynamic reconstructed images. The whole blood concentration was derived from a blood

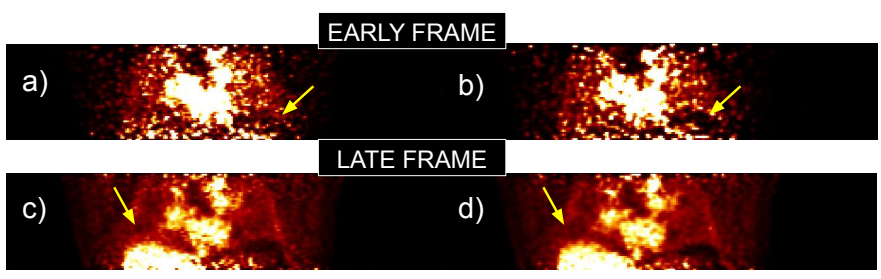

Fig. 2. Reconstructions from an early frame: a) standard OSEM, b) MCIR OSEM, and from a later frame: c) standard OSEM and c) MCIR OSEM. The yellow arrows mark positions where motion artefacts can visually be observed.
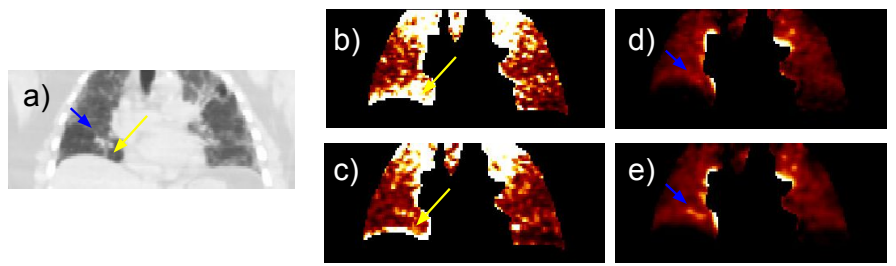

Fig. 3. a) CT image; residuals of the kinetic model fits from: b) standard OSEM, c) MCIR OSEM; $V_{\mathrm{B}}$ images: c) standard OSEM and d) MCIR OSEM, within the dilated lung mask. The yellow arrows show increased residuals near the diaphragm and the blue arrows show lung structures.

time activity curve for a region of interest drawn onto the Ascending Aorta and fitted to a predefined model [7].

\section{B. Evaluation Methods}

To assess the goodness of the motion estimation from JRM, non-AC OSEM reconstructions (6 iterations, 8 subsets) were performed on the respiratory gated data $\left\{\boldsymbol{g}_{\ell}\right\}$ (entire dynamic acquisition). The position of the diaphragm is then compared to its position as given by JRM, warping the JRM-reconstructed average image to end-expiration and endinspiration. The "residuals" in the lung (defined as the squared differences between the actual concentration and the estimated concentration from the model) are used to demonstrate the goodness of fit.

Additionally, when available, the warped $\mu$ maps (using the deformation fields from JRM) are visually assessed against gated $\mu$ maps from the CINE-CT acquisition.

\section{Results}

The MCIR images are generally sharper than the standard OSEM ones, with some misalignment artefacts (e.g. near the diaphragm) clearly reduced after motion correction. Examples for two different time frames are shown in Figure 2. The $V_{\mathrm{B}}$ and residual error images (in the lung) are shown in Figure 3. Visually we can see that the fit residuals are higher near the diaphragm, and that some structures from the CT image are more visible in the MCIR $V_{\mathrm{B}}$ image. We also observed that the mean residual in the lung was reduced by $18.7 \%$ after motion compensation in average for all acquisitions (result not showed here).

The JRM motion was compared visually and using profiles against gated non-AC reconstructions (Figure 4). We observe that the images were properly corrected for attenuation thanks to the realignment of $\boldsymbol{\mu}$ to the gated data with the motion $\varphi$. 


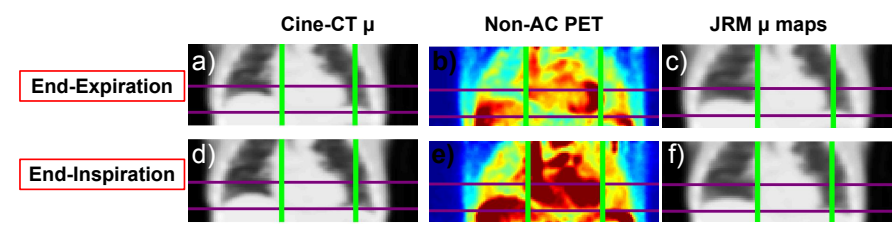

Fig. 5. End-Expiration: a) $\mu$ map from CINE-CT, b) non-AC, c) warped $\mu$ map; End-Inspiration: d) $\mu$ map from CINE-CT, e) non-AC, f) warped $\mu$ map.
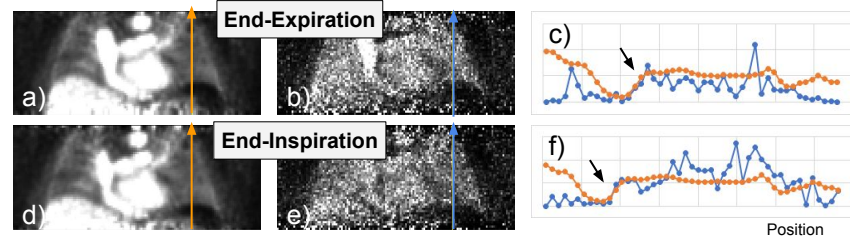

Fig. 4. Comparison of warped JRM images and non-AC gated reconstructions, along a Anterior-Posterior profile: at end-expiration: a) warped JRM, b) non$\mathrm{AC}, \mathrm{c})$ profiles, and at end-inspiration: d) warped JRM, e) non-AC, f) profiles. The black arrows show the matched diaphragm locations for the two gates.

Additionally for 4 acquisitions, CINE-CT acquisitions were available. One of the patients moved between the CINE-CT and the PET acquisitions (shift of approximately $1 \mathrm{~cm}$ ), producing conspicuous artefacts on the reconstructed images (ribs/around the heart). The method mostly realigned the $\mu$ map to the PET gates (as compared to the non-AC reconstructions), as shown in Figure 5. For the three other patients, the warped JRM $\mu$ maps were in good alignment with the CINE-CT $\mu$ maps. although the lung densities are not matching due to the expansion/dilation of the lungs [8].

\section{CONCLUSION AND Discussion}

The results of this work show that JRM can provide good estimates of motion for dynamic acquisitions of $\approx 15$-min total duration. The compartmental modelling on the motion corrected images had decreased residuals particularly near the diaphragm. This method, however, relies on negligible bulk motion during the PET acquisition and an accurate surrogate signal (no drift). This could be assessed by estimating the motion from 2 distinct subsets of the data. Jiao et al. 2017 [9] has shown promising results in direct joint compartmental modelling in the brain, approximating each dynamic frame as motion-free. This is however not the case in the lung, but an extended version of it could be possible with additional respiratory gates.

\section{REFERENCES}

[1] R. N. Gunn et al., "Positron emission tomography compartmental models: A basis pursuit strategy for kinetic modeling," Journal of Cerebral Blood Flow and Metabolism, vol. 22, no. 12, pp. 1425-1439, 2002.

[2] M. W. Jacobson et al., "Joint estimation of image and deformation parameters in motion-corrected PET," 4, vol. 5, 2003, pp. 3290-3294.

[3] A. Bousse et al., "Maximum-likelihood joint image reconstruction/motion estimation in attenuation-corrected respiratory gated PET/CT using a single attenuation map," IEEE Transactions on Medical Imaging, vol. 35, no. 1, pp. 217-228, 2016.

[4] V. Bettinardi et al., "Physical Performance of the new hybrid PET/CT Discovery-690," Medical Physics, vol. 38, no. 10, pp. 5394-5411, 2011.
[5] C. Chan et al., "Non-Rigid Event-by-Event Continuous Respiratory Motion Compensated List-Mode Reconstruction for PET," IEEE Transactions on Medical Imaging, vol. 37, no. 2, pp. 504-515, 2018.

[6] B. F. Holman, PhD thesis, University College London, London, UK, 2016.

[7] D. Feng et al., "A computer simulation study on the input function sampling schedules in tracer kinetic modeling with positron emission tomography (PET)," Computer Methods and Programs in Biomedicine, vol. 45, no. 3, pp. 175-186, 1994.

[8] V. Cuplov et al., "Issues in quantification of registered respiratory gated PET/CT in the lung," Physics in Medicine and Biology, vol. 63, no. 1, 2018.

[9] J. Jiao et al., "Direct Parametric Reconstruction with Joint Motion Estimation/Correction for Dynamic Brain PET Data," IEEE Transactions on Medical Imaging, vol. 36, no. 1, pp. 203-213, 2017. 